hep-th/0404101

CALT-68-2491

\title{
D Branes and Phases on String Worldsheet
}

\author{
Takuya Okuda and Hirosi Ooguri \\ California Institute of Technology, Pasadena, CA 91125, USA
}

\begin{abstract}
We generalize the worldsheet derivation of the topological open/closed string duality given in hep-th/0205297 to cases when there are different types of D branes on the open string side. We use the mirror Landau-Ginzburg description to clarify the correspondence between $\mathrm{D}$ branes on the open string side and $C$ phases on the closed string side. We also discuss the duality from the point of view of the B model.
\end{abstract}




\section{Introduction}

The large $N$ duality between open and close string theories has played important roles in recent development in string theory. The AdS/CFT correspondence [1] has provided insights into quantum gravity and strongly coupled gauge theories. The topological open/closed string duality [2] has uncovered relations between spectral density of random matrix models and geometry of Calabi-Yau manifolds and lead to the construction of matrix models to compute effective superpotential terms for supersymmetric gauge theories in four dimensions [3].

In the case of the topological string duality, the string coupling constant $g_{s}$ is the same for both open and closed string sides, and the 't Hooft couplings of the open string are identified with geometric moduli of the closed string side. Thus, it should be possible to give a microscopic explanation of the duality order by order in the string coupling expansion, namely from the point of view of the string worldsheet. (This should also be the case for the AdS/CFT correspondence when the coupling constant does not run.) In [4, the worldsheet derivation was given for the case when the open string side is defined on the cotangent space of a three-sphere $T^{*} S^{3}$ with $N$ D brane wrapping the base $S^{3}$. The closed string side is the resolved conifold whose Kähler modulus $t$, which is the size of the blown up $S^{2}$, is identified with the 't Hooft coupling $g_{s} N$ of the open string side. The strategy was to start with the closed string side and expand string amplitudes for small $t$. It has been noted in [5, 6] that in the linear sigma model description the closed string worldsheet at $t=0$ develops a new non-geometric phase - the $C$ phase - besides the geometric phase (Higgs phase) which flows to the non-linear sigma model for the resolved conifold in the IR limit. In [4, it was shown that the $C$ phase can contribute to closed string amplitudes only in the following two cases:

(1) Each domain in the $C$ phase has the topology of a disk.

(2) The entire worldsheet is in the $C$ phase.

Moreover it was found that each disk in the $C$ phase contributes a factor of $t$ to the amplitudes. By interpreting $C$ domains as holes on the worldsheet, the sum over disks in the $C$ phase reproduces the open string Feynman diagram expansion with $t$ being identified with the 't Hooft coupling. On the other hand, worldsheets that are entirely in the $C$ phase do not correspond to any open string diagrams. It was shown that the sum of such worldsheets to all order in the string coupling constant expansion correctly captures the gauge group volume vol $U(N)$ for the open string, which indeed does not come from Feynman diagrams but is needed to reproduce open string amplitudes.

Thus, this approach shows that the closed string on the resolved conifold has the open string expansion with the Kähler modulus $t$ being equal to the 't Hooft coupling on the open string side. One may be more ambitious and try to reproduce the D brane 
boundary condition. Some of the basic features of the boundary condition can been seen in this approach: at the interface of the $C$ phase and the geometric phase, one finds that the worldsheet is pulled toward the apex of the conifold where the $S^{2}$ shrinks to zero size at $t=0$ and the $S^{3}$ emerges after the conifold transition. However the precise boundary condition for the D branes wrapping the $S^{3}$ - for example, the Neumann boundary condition along the branes - has not been reproduced in this approach since the linear sigma model does not describe the geometry with $S^{3}$ of finite size. Although the size of $S^{3}$, being a complex structure modulus, is not relevant in the A-model and can be infinitesimal, it is desirable to understanding how D brane boundary conditions are reproduced from the closed string point of view.

In this paper, we will clarify the relation between $\mathrm{D}$ branes in open string and $C$ phases in closed string by studying cases in which there are several D branes on the open string side. We will consider the open string on the $\mathbf{Z}_{p}$ quotient of $T^{*} S^{3}$, whose base is the lens space $S^{3} / \mathbf{Z}_{p}$ [7]. Since the fundamental group of $S^{3} / \mathbf{Z}_{p}$ is $\mathbf{Z}_{p}$, there are $p$ different types of $\mathrm{D}$ branes whose holonomies along the homotopy generator are given by $e^{2 \pi i a / p}(a=0,1, \ldots, p-1)$. It turns out that the closed string dual has $p$ different $C$ phases on the worldsheet, and we show how each $C$ phase is identified with the corresponding D brane by studying the behavior of linear sigma model variables at the interface of each $C$ phase with the geometric phase. We find it is useful to use the Landau-Ginzburg B model which is T-dual to the linear sigma model [8] since much of the analysis in the B-model can be carried out at the classical level. We will also discuss how this can be seen from the point of view of the mirror manifold.

It is straightforward to apply the worldsheet derivation in [4] and in this paper to other toric Calabi-Yau manifolds which are known to have open string duals [9], for example the one described by $Q_{1}=(1,-1,0,1,-1)$ and $Q_{2}=(0,0,1,-2,1)$. It would be interesting to analyze closed string theories on more general toric manifolds and to discover new large $N$ dualities.

This paper is organized as follows. In section 2, we will review the worldsheet derivation of [4] in the conifold case. We will use the Landau-Ginzburg B-model to simplify some of the steps in the original derivation. We will then extend the analysis to the quotients of the conifold and explain the correspondence between $\mathrm{D}$ branes and $C$ phases from the Landau-Ginzburg description. In section 3, we will discuss the open/closed string duality as seen from the point of view of the mirror manifolds. 


\section{D branes and phases}

\subsection{Review of the conifold case}

In [2], it was conjectured that the A-type topological closed string theory on the resolved conifold with the Kähler modulus $t$ is equivalent to the A-type topological open string theory on the cotangent space $T^{*} S^{3}$ (or equivalently on the deformed conifold) with $N$ D branes wrapping the base $S^{3}$. Here the Kähler modulus $t$ of the closed string side is identified with the 't Hooft coupling $g_{s} N$ of the open string side, with the string coupling constant $g_{s}$ being the same on both sides. There have been several nontrivial checks of the conjecture [10, 11, 12, 13. Finally, a worldsheet derivation of the open/closed string duality was given in [4. In this subsection, we will review the derivation, using the mirror Landau-Ginzburg model [8] to simplify some of the steps.

The strategy is to start with the closed string side, expand string amplitudes around $t=0$, and show that a sum over open string Feynman diagrams emerges in the $t$ expansion. Since $t=0$ is a singular limit of the target space, it is useful to describe the worldsheet by the linear sigma model. The worldsheet theory consists of four chiral superfields $\Phi_{1}, \Phi_{2}, \Phi_{3}, \Phi_{4}$ with charges given by

$$
Q=(1,1,-1,-1),
$$

and a gauge multiplet coupled to $Q$. Following [5], we rearrange the gauge multiplet into a twisted chiral multiplet $\Sigma$. When $t$ is non-zero, there is a potential for the scalar field $\sigma$ which is the lowest component of $\Sigma$ and the linear sigma model flows to the nonlinear sigma model with the resolved conifold as the target space. In this description, the singularity at $t=0$ is characterized by the fact that the potential for $\sigma$ disappears in the limit and it can become indefinitely large without costing the worldsheet action - a new non-compact and non-geometric phase emerges on the worldsheet [5]. The idea of the derivation of the large $N$ duality, advocated in [2] and quantified in [4], is to regard domains in this new phase as holes on the worldsheet and phase boundaries as representing $\mathrm{D}$ branes.

The derivation of [4] can be streamlined by using the mirror of the linear sigma model, which is found by performing the $T$-dual transformation on phase rotations of $\Phi_{j}$, keeping $\Sigma$ as a spectator [8]. The $T$-dual of $\Phi_{j}$ are twisted chiral superfields $Y_{j}$ with periodicity $Y_{j} \sim Y_{j}+2 \pi i$. Combined with $\Sigma$, the mirror is a Landau-Ginzburg model with the superpotential $W$ given by

$$
W=\Sigma\left(Y_{1}+Y_{2}-Y_{3}-Y_{4}-t\right)-\sum_{j=1}^{4} e^{-Y_{j}} .
$$

Note that, since the original model is A-twisted, the mirror Landau-Ginzburg model is B-twisted. It is easy to check that, when $t$ is not equal to zero, there is no flat direction 
for the superpotential. It is believed that the Landau-Ginzburg model in this case flows to the mirror of the sigma model for the conifold. At $t=0$, the superpotential remains flat for

$$
\begin{aligned}
& y_{1}=y_{2}=-\log \sigma+\pi i \\
& y_{3}=y_{4}=-\log \sigma
\end{aligned}
$$

with $\sigma$ being arbitrary. Here $y_{i}$ is the lowest component scalar field in $Y_{i}$. Following [4], we call this flat direction the $C$ phase. ${ }^{1}$

In [4], it was argued that the $C$ phase is described by a Landau-Ginzburg model for a single variable $X$ with an effective superpotential

$$
W_{e f f}=-\frac{t}{X} .
$$

It is straightforward to derive this from the B-model. When $\sigma$ is large, the potential for $Y_{i}$ becomes steep and we can integrate them out using the Gaussian approximation. Since the mirror Landau-Ginzburg model is B-twisted, it is sufficient to consider an integral over constant maps,

$$
\int d Y_{1} d Y_{2} d Y_{3} d Y_{4} \exp (-W) \sim \frac{1}{\Sigma^{2}} \exp (t \Sigma) .
$$

The pre-factor $1 / \Sigma^{2}$ gives a non-canonical measure for $\Sigma$. We can absorb it by changing the variable $\Sigma \rightarrow X=\Sigma^{-1}$. This gives the effective superpotential (2.4) for the canonically normalized variable $X$.

We found that there are two phases in the worldsheet theory: the geometric phase, which flows in the IR to the non-linear sigma model on the conifold, and the $C$ phase, which is non-geometric and is described by the effective Landau-Ginzburg model with the superpotential $W_{e f f}=-t / X$. The functional integral then includes a sum over domains in the $C$ phase on the worldsheet. Let us state the following two facts that were shown in [4]:

(1) A domain in the $C$ phase contributes to a topological string amplitude only if (a) the domain has the topology of a disk or (b) the entire worldsheet is in $C$ phase.

(2) Each $C$ domain of the disk topology contributes to the amplitude by a factor of $t$. This follows from the integral

$$
\oint d X e^{t / X}=2 \pi i t .
$$

\footnotetext{
${ }^{1}$ This is called the $C$ phase in order to distinguish it from the Coulomb phase of the model, which is decoupled from the geometric phase in the IR limit. For a more detailed specification of the $C$ phase, see [4].
} 
Thus, if we regard each $C$ domain of the disk topology as a hole on the worldsheet, the closed topological string amplitude is expressed as a sum of open topological amplitudes with a boundary of each hole being weighted with a factor of $t$.

When the entire worldsheet is in the $C$ phase, the closed topological string amplitude at genus $g$ is given by $\chi\left(\mathcal{M}_{g}\right) / t^{2 g-2}$, where $\chi\left(\mathcal{M}_{g}\right)$ is the Euler characteristic of the moduli space of genus $g$ surfaces and is equal to $B_{2 g} / 2 g(2 g-2)$. The negative power in $t$ (for $g \geq 2$ ) reflects the singularity at $t \rightarrow 0$. Summing this up over all genera, one obtains [4]

$$
\sum_{g} \chi\left(\mathcal{M}_{g}\right)\left(\frac{g_{s}}{t}\right)^{2 g-2}=\sum_{g} \frac{B_{2 g}}{2 g(2 g-2) N^{2 g-2}}=\log \operatorname{vol} U(N),
$$

where we used $t=g_{s} N$. Thus, the sum over the worldsheet in the pure $C$ phase gives the gauge volume factor vol $U(N)$ for the gauge theory.

This establishes that the closed topological string theory on the resolved conifold is equivalent to some open topological string theory, with gauge group $U(N)$ and 't Hooft coupling $t$. We have not yet shown on which D branes open strings are ending. According to the conjecture [2], the D branes should be wrapping the base $S^{3}$ of the deformed conifold. To see how the boundary condition emerges from the closed string dual, we first note that the transition from the resolved conifold to the deformed conifold is a local operation near the conifold singularity. Thus, away from the base $S^{3}$, we can approximate the deformed conifold by the geometric phase (Higgs phase) of the linear sigma model for the resolved conifold. Since A-model amplitudes are independent of the complex structure, we can make the size of $S^{3}$ as small as we like, making the approximation increasingly accurate. In the $C$ phase, all the $\Phi^{i}$ fields in the sigma model become massive. Thus, $\Phi^{i} \rightarrow 0$ as we approach the "hole" on the closed string worldsheet, and it is roughly where the base $S^{3}$ is located. Reproducing the precise boundary condition for D branes wrapping $S^{3}$ - for example, deriving the Neumann boundary condition along $S^{3}$ - is difficult in this approach since the linear sigma model does not describe the geometry of the deformed conifold with finite $S^{3}$.

Although reproducing a precise boundary condition for each D brane may be difficult, one may ask if we can distinguish different types of D branes in this approach. In the following subsections, we will demonstrate that it is possible.

\subsection{Gauge theory on $S^{3} / \mathrm{Z}_{2}$}

As the first example in which there are more than one types of $\mathrm{D}$ branes, we consider the Chern-Simons gauge theory on the lens space $S^{3} / \mathbf{Z}_{2}$. Classical solutions are flat connections, which are labeled by holonomy matrices for the homotopy generator of the space. Since the fundamental group is $\mathbf{Z}_{2}$ in this case, the $U(N)$ gauge theory 
can have a holonomy matrix with $N_{1}$ eigenvalues being $(+1)$ and $N_{2}$ eigenvalues being (-1), where $N=N_{1}+N_{2}$. This breaks the gauge group to $U\left(N_{1}\right) \times U\left(N_{2}\right)$. This can be realized by considering the topological string theory on $T^{*} S^{3} / \mathbf{Z}_{2}$, with $N_{1} \mathrm{D}$ branes wrapping the lens space with the trivial bundle and $N_{2}$ D branes wrapping the same space with the bundle twisted by $(-1)$.

According to the conjecture in [7, the target space of the closed string dual is the $\mathbf{Z}_{2}$ quotient of the resolved conifold. This space has two Kähler moduli, which are naturally identified with the two 't Hooft couplings $g_{s} N_{1}$ and $g_{s} N_{2}$ of the open string. This conjecture has also been tested in nontrivial ways [7, 14, 15].

The closed string worldsheet is described by a linear sigma model with five chiral multiplets $\Phi_{i}, i=0, \ldots, 4$ with two sets of charges

$$
\begin{aligned}
& Q_{1}=(-2,1,1,0,0), \\
& Q_{2}=(-2,0,0,1,1),
\end{aligned}
$$

and two gauge multiplets coupled to these charges. Since $Q_{1}-Q_{2}=(0,1,1,-1,-1)$, solving the $\mathrm{D}$ term constraint and dividing by the $U(1)$ gauge symmetry coupled to this combination of charges reduce $\phi_{1}, \ldots, \phi_{4}$ to the resolved conifold. In the cone $r_{1}<0, r_{1}-r_{2}<0$, since $\phi_{0} \neq 0$, we can use the remaining $Q_{1}$ gauge symmetry to fix the phase of $\phi_{0}$. This leaves out a residual $\mathbf{Z}_{2}$ gauge symmetry acting as

$$
\left(\phi_{0}, \phi_{1}, \phi_{2}, \phi_{3}, \phi_{4}\right) \rightarrow\left(\phi_{0},-\phi_{1},-\phi_{2}, \phi_{3}, \phi_{4}\right) \text {. }
$$

Thus we find the $\mathbf{Z}_{2}$ quotient of the resolved conifold as the target space.

As in the previous subsection, we rearrange the gauge multiplets into twisted chiral superfields, $\Sigma_{1}$ and $\Sigma_{2}$, and perform the $T$-dual transformation along phase rotations of the five chiral superfields to arrive at the B-twisted Landau-Ginzburg model with the superpotential,

$$
W=\Sigma_{1}\left(-2 Y_{0}+Y_{1}+Y_{2}-t_{1}\right)+\Sigma_{2}\left(-2 Y_{0}+Y_{3}+Y_{4}-t_{2}\right)-\sum_{i=0}^{5} e^{-Y_{i}},
$$

where the two Kähler moduli, $t_{1}$ and $t_{2}$, are linearly coupled to the gauge multiplets.

Let us examine when this superpotential has flat directions. Solving $\partial W / \partial y_{i}=0$, we find

$$
\begin{aligned}
& y_{0}=-\log \left(2 \sigma_{1}+2 \sigma_{2}\right), \\
& y_{1}=y_{2}=-\log \left(-\sigma_{1}\right), \\
& y_{3}=y_{4}=-\log \left(-\sigma_{2}\right) .
\end{aligned}
$$

By substituting this into the remaining equations,

$$
\frac{\partial W}{\partial \sigma_{a}}=-2 y_{0}+y_{a}+y_{a+1}-t_{a}=0 \quad(a=1,2),
$$


we find

$$
\begin{aligned}
& e^{t_{1}}=4\left(1+\frac{\sigma_{2}}{\sigma_{1}}\right)^{2} \\
& e^{t_{2}}=4\left(1+\frac{\sigma_{1}}{\sigma_{2}}\right)^{2}
\end{aligned}
$$

Since both $t_{1}$ and $t_{2}$ depend only on the ratio $\sigma_{1} / \sigma_{2}$, this is possible only if they satisfy the relation

$$
\Delta=16\left(e^{-t_{1}}-e^{-t_{2}}\right)^{2}-8\left(e^{-t_{1}}+e^{-t_{2}}\right)+1=0,
$$

which is obtained by eliminating $\sigma_{1} / \sigma_{2}$ from the two equations in (2.13). The subspace of the Kähler moduli space where $\Delta=0$ is known as the singular locus ${ }^{2}$. If the Kähler moduli satisfy $\Delta=0$, the superpotential has a flat direction corresponding to the scaling of $\sigma_{1}$ and $\sigma_{2}$ while keeping their ratio fixed.

This model has two different $C$ phases. For a generic point on the singular locus, only one of the two $C$ phases emerges. But there is a particular point where both co-exist. Let us consider the limit $^{3}$

$$
t_{1}, t_{2} \rightarrow-\infty, \quad t_{1}-t_{2} \rightarrow 0
$$

In this limit, the condition (2.14) for the singular locus gives

$$
t_{1}-t_{2}= \pm e^{t_{1} / 2}+O\left(e^{t_{1}}\right)
$$

For such $t_{1}, t_{2}$, we can solve (2.13) as

$$
\sigma_{2}=-\sigma_{1} \pm e^{t_{1} / 2} \sigma_{1}+O\left(e^{t_{1}}\right) .
$$

\footnotetext{
${ }^{2}$ The singular locus can also be derived from the linear sigma model point of view [16. In this case, we have to take into account quantum corrections in the linear sigma model. This is in contrast to the mirror Landau-Ginzburg description, where the singular locus (2.14) is derived from the classical analysis of the superpotential (2.10).

${ }^{3}$ This limit is motivated by the fact that the two $C$ phases co-exist as we will show below. A geometric motivation for the limit will be made clear in section 3 .
} 
Substituting this into (2.11), we find two flat directions

$$
\begin{array}{ll}
C_{+} \text {phase }: & \sigma_{1}=-\sigma_{2}=\sigma, \\
& y_{0}=-\frac{t_{1}}{2}-\log \sigma, \\
& y_{1}=y_{2}=-\log \sigma, \\
& y_{3}=y_{4}=-\log \sigma+\pi i . \\
C_{-} \text {phase }: \quad & \sigma_{1}=-\sigma_{2}=\sigma, \\
& y_{0}=-\frac{t_{1}}{2}-\log \sigma+\pi i, \\
& y_{1}=y_{2}=-\log \sigma, \\
& y_{3}=y_{4}=-\log \sigma+\pi i .
\end{array}
$$

Both are complex one-dimensional in the seven-dimensional space of $(\sigma, y)$ and are parametrized by $\sigma$. Note that the two phases are distinguished by the value of $y_{0}$. When $e^{t_{1}}$ and $e^{t_{2}}$ are small but finite, either $C_{+}$or $C_{-}$solves $d W=0$ depending on the sign $( \pm)$ on the right-hand side of (2.16). In the limit (2.15), both phases co-exist.

In this model, the flat coordinates $\hat{t}_{+}, \hat{t}_{-}$are non-linear functions of the parameters $t_{1}, t_{2}$ in the superpotential (2.10). They can be computed either by the integrals $\int d \sigma d y e^{-W}$ with different choice of contours or by going to the mirror of the $\mathbf{Z}_{2}$ quotient of the resolved conifold and performing period integrals. From the latter point of view, $\hat{t}_{1}$ and $\hat{t}_{2}$ are periods of two 3 -cycles in the mirror manifold. We will discuss the latter point of view in more detail in section 3. In terms of the flat coordinates, the condition $\Delta=0$ is equivalent to $\hat{t}_{+}=0$ or $\hat{t}_{-}=0$, where one of the two $C$ phases emerges. The limit (2.15) corresponds to $\hat{t}_{+}=0$ and $\hat{t}_{-}=0$, consistently with the fact that both $C$ phases are realized in the limit.

Let us examine the limit more closely. The flat coordinates are expressed in the limit as

$$
\begin{aligned}
& \hat{t}_{+}=e^{t_{1} / 2}+t_{1}-t_{2}+O\left(e^{t_{1}}\right) \\
& \hat{t}_{-}=-e^{t_{1} / 2}+t_{1}-t_{2}+O\left(e^{t_{1}}\right) .
\end{aligned}
$$

Comparing this with (2.16), we find that the $C_{+}\left(C_{-}\right)$phase emerges at $\hat{t}_{+}=0$ (at $\left.\hat{t}_{-}=0\right)$. The two $C$ phases co-exist when both flat coordinates vanish. The two flat coordinates are exchanged as $\left(t_{1}, t_{2}\right) \rightarrow\left(t_{1}+2 \pi i, t_{2}+2 \pi i\right)$ and at the same time the two $C$ phases are also exchanged.

Thus, at $\hat{t}_{+}=\hat{t}_{-}=0$, both $C$ phases as well as the geometric phase co-exist on the worldsheet. We claim that the two $C$ phases correspond to the two types of $\mathrm{D}$ branes with different holonomies around the homotopy generator $\gamma$ of $S^{3} / Z_{2}$. This can be shown in the following three steps: 
(1) We first note that $C_{+}$and $C_{-}$are distinguished by the values of $y$ as in (2.18) and (2.19), and that they are related to each other by a shift of $y_{0}$ by $\pi i$, one half of the periodicity of $y_{0}$.

(2) Since a shift of $y_{i}$ in the imaginary direction is $T$-dual to a phase rotation of $\phi_{i}$ for each $i=0, \ldots, 5$, the $C$ branches represent $\mathrm{D}$ branes wrapping around the phase rotations of $\phi$ 's. Since $C_{+}$and $C_{-}$differ by the shift of one half of the period of $y_{0}$, the corresponding two types of $\mathrm{D}$ branes in term of the dual $\phi$ variables are related to each other by a multiplication of $(-1)$ to the holonomy of the gauge field around a $2 \pi$ phase rotation of $\phi_{0}$.

(3) What remains is to identify this $(-1)$ as the relative holonomy around the homotopy generator $\gamma$ on the $\mathrm{D}$ brane worldvolume $S^{3} / \mathbf{Z}_{2}$. Since the fundamental groups of $S^{3} / \mathbf{Z}_{2}$ and $T^{*} S^{3} / \mathbf{Z}_{2}$ are isomorphic and since the conifold transition is a local operation near the singularity, we can lift $\gamma$ from the base and describe it in the linear sigma model variables. To see that $\gamma$ is homotopic to the $2 \pi$ phase rotation of $\phi_{0}$, we just have to note that the latter is gauge equivalent via the $Q_{1}$ gauge transformation to the $\pi$ rotation,

$$
\left(\phi_{0}, e^{i \theta} \phi_{1}, e^{i \theta} \phi_{2}, \phi_{3}, \phi_{4}\right), \quad 0 \leq \theta \leq \pi
$$

and that this path is closed because of the $\mathbf{Z}_{2}$ quotient described in the third paragraph of this subsection.

We have established that the two $C$ phases emerge in the limit $\hat{t}_{+}, \hat{t}_{-} \rightarrow 0$. The boundary conditions at the interface of the $C$ phases and the geometric phase are related to each other by the shift of $\pi i$ of the value of $y_{0}$. Via the $T$-duality, they are mapped to boundary conditions on the linear sigma model variables related to each other by a multiplication of $(-1)$ to the holonomies around the homotopy generator of $S^{3} / \mathbf{Z}_{p}$, i.e. the two types of $\mathrm{D}$ branes expected in the open string dual. ${ }^{4}$

For the same reason as in the case of the conifold discussed in [4] and reviewed in the last subsection, $C$ domains contribute to topological string amplitudes only if they are of the disk topology or if they cover the entire worldsheet.

The large $N$ duality conjecture states that the 't Hooft couplings for the two types of $\mathrm{D}$ branes are given by the flat coordinate $\hat{t}_{ \pm}$on the closed string side. This can be shown as follows. Each of the $C$ branches is complex one-dimensional parametrized by $\sigma$ in (2.18) and (2.19). An integral in the direction transverse to $\sigma$ imposes one linear constraint on the five $Y$ fields. For large value of $\sigma$, remaining four $Y$ fields can be integrated out in the Gaussian approximation. As in the conifold case, this results in a

\footnotetext{
${ }^{4}$ Since the open string is in the adjoint representation of the gauge group, only the relative holonomy of the two types of $\mathrm{D}$ branes has an invariant meaning. This is $T$-dual to the fact that only the relative value of $y_{0}$ in the two $C$ phases is relevant because of the translational invariance. In fact gauge theory amplitudes are invariant under exchange of $N_{1}$ and $N_{2}$, the numbers of the two types of D branes [7].
} 
superpotential linear in $\Sigma$ with the non-canonical measure of $1 / \Sigma^{2}$. Using the variable $X=1 / \Sigma$, one finds an effective superpotential $\sim 1 / X$ with the canonical measure. To find the coefficient of the $1 / X$ potential, we note the following two well-known facts.

(1) The genus- $g$ closed string amplitude for the Landau-Ginzburg model with the superpotential $W=t / X$ is proportional to $t^{2-2 g}$ [17].

(2) The singular part of the genus- $g$ closed string amplitude for small $\hat{t}_{ \pm}$is proportional to $\hat{t}_{ \pm}^{2-2 g}[18$.

Note that both statements follow from studies on the closed string side and do not assume the open/closed string duality. Comparing them, we find that the effective superpotential in the $C_{ \pm}$phase is given by $\hat{t}_{ \pm} / X$ respectively. The disk amplitude is then computed exactly as in the conifold case, giving rise to the factor $\hat{t}_{ \pm}$for the $C_{ \pm}$ phase. This is what we wanted to show.

\subsection{Gauge theory on $S^{3} / \mathrm{Z}_{p}$}

It is straightforward to generalize the result in the previous subsection to the case of the $\mathbf{Z}_{p}$ quotient of the conifold for $p \geq 2$. In this case, the conjectured gauge theory dual is on the lens space $S^{3} / \mathbf{Z}_{p}\left[\mathbf{7}\right.$. Since the fundamental group of the space is $\mathbf{Z}_{p}$, there are $p$ different types of $\mathrm{D}$ branes whose holonomies around the homotopy generator are given by $e^{2 \pi i a / p}(a=0,1, \ldots, p-1)$. We would like to see how they are identified with $p$ different $C$ phases in the closed string dual.

The worldsheet of the closed string on the $\mathbf{Z}_{p}$ quotient of the resolved conifold can be described by the linear sigma model with $(p+3)$ chiral fields $\Phi_{0}, \Phi_{1}, \ldots, \Phi_{p+2}$ coupled to $p$ gauge fields with the following charge vectors [15],

$$
\begin{aligned}
& \Phi_{0}, \quad \Phi_{1}, \quad \Phi_{2}, \quad \Phi_{3}, \quad \Phi_{4}, \quad \Phi_{5}, \ldots, \quad \Phi_{4+j}, \cdots, \Phi_{p+2}
\end{aligned}
$$

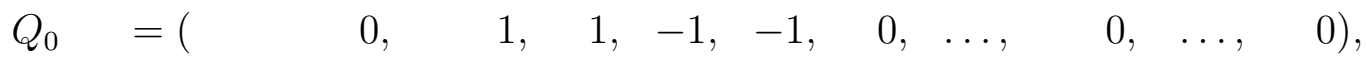

$$
\begin{aligned}
& Q_{j}=\left(\begin{array}{llllllllll}
-(j+1), & j, & 0, & 0, & 0, & 0, & \ldots, & 1, & \ldots, & 0
\end{array}\right), \\
& Q_{p-1}=(\quad-p, p-1, \quad 1, \quad 0, \quad 0, \quad 0, \ldots, \quad \ldots, \quad \ldots, \quad 0) \text {, }
\end{aligned}
$$

where $j=1, \cdots, p-2$. Let us show that that this indeed describes the $\mathbf{Z}_{p}$ quotient of the resolved conifold in the cone,

$$
\begin{aligned}
& r_{0}<0, \\
& r_{p-1}<0, \\
& -r_{j}+\frac{j+1}{p} r_{p-1}<0 \quad(1 \leq j \leq p-2) .
\end{aligned}
$$


We write $U(1)_{a}$ for the $U(1)$ generated by $Q_{a}$, and the corresponding $\mathrm{D}$ term is $D_{a}:=$ $\sum_{i} Q_{a i}\left|\phi_{i}\right|^{2}-r_{a}(a=1, \ldots, p-1)$. If $r_{0}<0,\left\{D_{0}=0\right\} / U(1)_{0}$ describes the resolved conifold. If $r_{p-1}<0, D_{p-1}=0$ does not allow $\phi_{0}$ to vanish. Thus, we can use the $U(1)_{p-1}$ gauge symmetry to fix the phase of $\phi_{0}$. Since $\phi_{0}$ carries $(-p)$ units of $Q_{p-1}$, there is a $\mathbf{Z}_{p}$ residual gauge symmetry of $U(1)_{p-1}$ given by

$$
\left(\phi_{0}, \phi_{1}, \phi_{2}, \phi_{3 \leq i \leq p-1}\right) \rightarrow\left(\phi_{0}, e^{-\frac{2 \pi i}{p}} \phi_{1}, e^{\frac{2 \pi i}{p}} \phi_{2}, \phi_{3 \leq i \leq p-1}\right) .
$$

Other gauge groups $U(1)_{1 \leq j \leq p-2}$ are completely fixed if $-r_{j}+\frac{j+1}{p} r_{p-1}<0$ since $0=$ $D_{j}-\frac{j+1}{p} D_{p-1}=\left(\frac{j-p+1}{p}\right)\left|\phi_{1}\right|^{2}-\frac{j+1}{p}\left|\phi_{2}\right|^{2}+\left|\phi_{4+j}\right|^{2}-r_{j}+\frac{j+1}{p} r_{p-1}$ requires $\phi_{4+j}$ to be nonzero. Thus, we obtain the $\mathbf{Z}_{p}$ quotient of the resolved conifold as a space of solutions to the $\mathrm{D}$ term constraints up to gauge transformations.

The mirror Landau-Ginzburg model has the superpotential

$$
\begin{aligned}
W= & \Sigma_{0}\left(Y_{1}+Y_{2}-Y_{3}-Y_{4}-t_{0}\right)+\sum_{j=1}^{p-2} \Sigma_{j}\left(-(j+1) Y_{0}+j Y_{1}+Y_{4+j}-t_{j}\right) \\
& +\Sigma_{p-1}\left(-p Y_{0}+(p-1) Y_{1}+Y_{2}-t_{p-1}\right)-\sum_{j=0}^{p-1} e^{-Y_{j}}
\end{aligned}
$$

As in the previous subsection, we look for flat directions of the potential. Solving $\partial W / \partial y_{i}=0(i=0, \ldots, p+2)$ gives

$$
\begin{aligned}
y_{0} & =-\log \left(\sum_{j=1}^{p-1}(j+1) \sigma_{j}\right) \\
y_{1} & =-\log \left(-\sigma_{0}-\sum_{j=1}^{p-1} j \sigma_{j}\right) \\
y_{2} & =-\log \left(-\sigma_{0}-\sigma_{p-1}\right) \\
y_{3} & =-\log \sigma_{0} \\
y_{4} & =-\log \sigma_{0} \\
y_{4+i} & =-\log \left(-\sigma_{i}\right), \quad(i=1, \ldots, p-2) .
\end{aligned}
$$

Substituting them into $\partial W / \partial \sigma_{a}=0(a=0, \ldots, p-1)$ gives $p$ relations of the form,

$$
\begin{aligned}
e^{t_{0}} & =\frac{\sigma_{0}^{2}}{\left(\sigma_{0}+\sigma_{p-1}\right)\left(\sigma_{0}+\sum_{j} j \sigma_{j}\right)} \\
e^{t_{k}} & =\frac{\left(-\sum_{j}(j+1) \sigma_{j}\right)^{k+1}}{\sigma_{k}\left(\sigma_{0}+\sum_{j} j \sigma_{j}\right)^{k}} \\
e^{t_{p-1}} & =\frac{\left(-\sum_{j}(j+1) \sigma_{i}\right)^{p}}{\left(\sigma_{0}+\sigma_{p-1}\right)\left(\sigma_{0}+\sum_{j} j \sigma_{j}\right)^{p-1}},
\end{aligned}
$$


where $k=1, \ldots, p-2$. Note that the right-hand sides are functions of the ratios of $\sigma$ 's. Since there are $p$ relations for $(p-1)$ variables, there is no solution for generic values of $t$ and thus no flat direction for $W$. The singular locus, where a flat direction emerges, is determined by eliminating $\sigma_{1} / \sigma_{0}, \ldots, \sigma_{p-1} / \sigma_{0}$ from these $p$ equations. The analysis of each of $C$ phase can be done as in the $\mathbf{Z}_{2}$ case. For example, since each $C$ phase is complex one-dimensional parametrized by the scaling of $\sigma$ 's, the functional integral over $\sigma$ in the transverse direction imposes $(p-1)$ linear constraints on $(p+3) Y$ variables, leaving 4 linear combinations of $Y$ free. The functional integrals of these 4 fields can be done in the Gaussian approximation, giving rise to the effective Landau-Ginzburg model with the $1 / X$ superpotential.

To understand how $p$ different $C$ phases emerge, it is useful to make the following change of variables,

$$
\begin{aligned}
Y_{0}^{\prime} & :=Y_{0}+\frac{t_{p-1}}{p}, \\
Y_{2}^{\prime}: & =-p Y_{0}+(p-1) Y_{1}+Y_{2}-t_{p-1}, \\
Y_{4}^{\prime}: & =-Y_{1}-Y_{2}+Y_{3}+Y_{4}+t_{0}, \\
Y_{4+j}^{\prime}: & =-(j+1) Y_{0}+j Y_{1}+Y_{4+j}-t_{j},
\end{aligned}
$$

so that the superpotential takes the form,

$$
\begin{aligned}
W & =-\Sigma_{0} Y_{4}^{\prime}+\sum_{j=1}^{p-2} \Sigma_{j} Y_{4+j}^{\prime}+\Sigma_{p-1} Y_{2}^{\prime}-\left(e^{\frac{t_{p-1}}{p}} e^{-Y_{0}^{\prime}}+e^{-Y_{1}}+e^{-Y_{2}^{\prime}-p Y_{0}^{\prime}+(p-1) Y_{1}}\right. \\
& \left.+e^{-Y_{3}}+e^{-Y_{4}^{\prime}-2 Y_{2}^{\prime}-p Y_{0}+(p-2) Y_{1}+Y_{3}+t_{0}}+\sum_{j=1}^{p-2} e^{-Y_{4+j}^{\prime}+\frac{j+1}{p} t_{p-1}-(j+1) Y_{0}^{\prime}+j Y_{1}-t_{j}}\right) .
\end{aligned}
$$

In the limit

$$
\begin{aligned}
& t_{0} \rightarrow 0, \\
& t_{p-1} \rightarrow-\infty, \\
& \frac{j+1}{p} t_{p-1}-t_{j} \rightarrow-\infty,
\end{aligned}
$$

the superpotential becomes

$$
\begin{aligned}
W= & -\Sigma_{0} Y_{4}^{\prime}+\sum_{j=1}^{p-2} \Sigma_{j} Y_{4+j}^{\prime}+\Sigma_{p-1} Y_{2}^{\prime}-\left(e^{-Y_{1}}+e^{-Y_{2}^{\prime}-p Y_{0}^{\prime}+(p-1) Y_{1}}\right. \\
& \left.+e^{-Y_{3}}+e^{-Y_{4}^{\prime}-2 Y_{2}^{\prime}-p Y_{0}^{\prime}+(p-2) Y_{1}+Y_{3}}\right) .
\end{aligned}
$$


Extremizing $W$ leads to $p$ different families of solutions

$$
\begin{array}{ll}
C_{k} \text { phase }: \quad & y_{0}^{\prime}=-\log \left(-\sigma_{0}\right)+\frac{2 \pi i}{p} k, \\
& y_{1}=-\log \left(-\sigma_{0}\right), \quad y_{2}^{\prime}=0 \\
& y_{3}=-\log \left(\sigma_{0}\right), \quad y_{4}^{\prime}=0 \\
& y_{4+j}^{\prime}=0, \quad \sigma_{j}=0 \quad(1 \leq j \leq p-2),
\end{array}
$$

where $k=0,1, \ldots, p-2$. We found that $p$ different $C$ phases co-exist in the limit (2.30).

The $p$ different $C$ phases are related to each other as $C_{k} \rightarrow C_{k+1}$ under the shift $y_{0}^{\prime} \rightarrow y_{0}^{\prime}+2 \pi i / p$. In terms of the original $y$ variables, the shift is expressed as

$$
\begin{aligned}
& \left(y_{0}, y_{1}, y_{2}, y_{3}, y_{4}, y_{4+j}\right) \\
& \quad \rightarrow\left(y_{0}+\frac{2 \pi i}{p}, y_{1}, y_{2}, y_{3}, y_{4}, y_{4+j}+\frac{2 \pi i}{p}(j+1)\right) .
\end{aligned}
$$

As explained in the second paragraph of this subsection, the homotopy generator of $S^{3} / \mathbf{Z}_{p}$ is the path

$$
\left(\phi_{0}, e^{-i \theta} \phi_{1}, e^{i \theta} \phi_{2}, \phi_{3}, \ldots\right), \quad 0 \leq \theta \leq \frac{2 \pi}{p} .
$$

Note that a $2 \pi$ rotation of $\phi_{1}$, keeping other variables fixed, is contractible even if one is away from the apex of the conifold since $\phi_{1}$ can vanish while maintaining the $\mathrm{D}$ term constraints. Thus, the $(-2 \pi / p)$ rotation of $\phi_{1}$ in the above can be replaced by the $2 \pi(p-1) / p$ rotation. Under the $U(1)_{p-1}$ gauge transformation with respect to the charge vector $Q_{p-1}$ in (2.22), this is gauge equivalent to a $2 \pi$ phase rotation of $\phi_{0}$. Since the map from $C_{k}$ to $C_{k+1}(k=0, \ldots, p-1)$ involves the $2 \pi i / p$ shift of $y_{0}$, their holonomies under the $2 \pi$ phase rotation of $\phi_{0}$ differ by $e^{2 \pi i / p}$. Namely, the relative holonomy of $C_{k}$ and $C_{k+1}$ around the homotopy generator of $S^{3} / \mathbf{Z}_{p}$ is equal to $e^{2 \pi i / p}$, precisely reproducing the large $N$ duality stated in the first paragraph of this subsection.

In principle, we can carry out the analysis further in this approach and find the flat coordinates explicitly in the Landau-Ginzburg description. However, it is more convenient and geometrically more intuitive to use the mirror manifold, which can be obtained by partially performing the functional integral of the Landau-Ginzburg model and making some change of variables [8]. We are going to discuss it in the next section.

\section{B-model large $N$ dualities}

The large $N$ duality from the point of view of the B-model has lead to the discovery of the relation between spectral density of matrix models and Calabi-Yau geometry. In 
this section, we will give a worldsheet derivation of this and related dualities.

\subsection{Mirror of the $\mathrm{Z}_{p}$ quotient of the resolved conifold}

In the last section, we considered the $\mathbf{Z}_{p}$ quotient of the resolved conifold. Here we will study the same problem but from the point of view of its mirror manifold. The mirror Calabi-Yau manifold is given by the equation [15],

$$
\begin{aligned}
0 & =G\left(x_{1}, x_{2}, u, v\right) \\
& \equiv x_{1}^{2}+x_{2}^{2}+\left(e^{v}-1\right)\left(e^{p u+v}-1\right)+e^{t_{0}}-1-e^{t_{p-1} / p+u+v}-\sum_{j=1}^{p-2} e^{\frac{j+1}{p} t_{p-1}-t_{j}+(j+1) u+v} .
\end{aligned}
$$

The non-linear sigma model on the above non-compact Calabi-Yau can be realized as the IR limit of the Landau-Ginzburg model with chiral superfields $\Lambda, X_{1}, X_{2}, U, V$ and the superpotential

$$
W=\Lambda G\left(X_{1}, X_{2}, U, V\right),
$$

where the scalar components of $U$ and $V$ are defined modulo $2 \pi i$. Indeed, as long as the geometry is smooth so that there is no solution to $G=d G=0$, the only solutions to $d W=0$ are $\Lambda=G=0$. Excitations transverse to $\Lambda=G=0$ are massive. Hence in the low-energy the theory flows to the non-linear sigma model on the geometry $G=0$. Such a Landau-Ginzburg model was considered in [6] in the case of deformed conifold. This Landau-Ginzburg model is related to the model used in subsection 2.3 by partially carrying out the functional integral and by making change of variables [8]. We will show that the worldsheet phase structure found in subsection 2.3 can also be obtained from this Landau-Ginzburg model.

In the limit

$$
\begin{aligned}
& t_{0} \rightarrow 0, \\
& t_{p-1} \rightarrow-\infty \\
& \frac{j+1}{p} t_{p-1}-t_{j} \rightarrow-\infty,
\end{aligned}
$$

we have

$$
G \sim x_{1}^{2}+x_{2}^{2}+\left(e^{p u+v}-1\right)\left(e^{v}-1\right)
$$

and the geometry $G=0$ develops $p$ conifold singularities at

$$
\begin{aligned}
\left(x_{1}, x_{2}, u, v\right) & =\left(0,0, \frac{2 \pi i}{p} a, 0\right), \\
a & =0,1, \ldots, p-1 .
\end{aligned}
$$


The singularities of the geometry are reflected in the worldsheet theory as the appearance of new non-compact directions in the space of zero-energy configurations. Namely, the worldsheet theory develops $p$ new flat directions where $\lambda$, the lowest component of $\Lambda$, is large while $\left(x_{1}, x_{2}, u, v\right)$ are fixed to the locations of conifold singularities.

We see that $\Lambda$ plays the same role as the $\Sigma$ field and $C$ phases can be defined as flat directions where $\lambda$ becomes large. Different $C$ phases are distinguished by the values of $u$. In each $C$ domain, since $\lambda$ is large, we can integrate out $x_{1}, x_{2}, u$ and $v$ by Gaussian approximation which produces the measure $d \lambda / \lambda^{2}$. Thus, the effective Landau-Ginzburg model of each of the $C$ phases is again with the $1 / X$ superpotential. The coefficient of the superpotential is given by the flat coordinate $\hat{t}_{a}(a=0, \ldots, p-1)$ since each $C$ phase is associated with shrinking of one of $p 3$-cycles.

In the language of the present subsection it is easy to generalize the analysis of the singular locus and the periods. Let us introduce new parameters $d_{0}, d_{1}, \ldots, d_{p-1}$ as functions of $t_{0}, \ldots, t_{p-1}$ so that $G$ is expressed as

$$
G=x_{1}^{2}+x_{2}^{2}+\left(e^{p u+v}-1\right)\left(e^{v}-1\right)+d_{0}+\sum_{j=1}^{p-1} d_{j} e^{j u+v} .
$$

The limit (3.37) is equivalent to $d_{j} \rightarrow 0$ for $j=0,1, \ldots, p-1$. Let us evaluate $\Delta$ and the periods $\hat{t}_{a}$ in this limit. Suppose $(u, v)$ are near $(2 \pi i a / p, 0)$ and write $(u, v)=$ $(2 \pi i a / p+\delta u, \delta v)$. Assuming that $\delta u$ and $\delta v$ are of the order $\mathcal{O}(d)$, we can expand $G$ to the quadratic order in the variation and find

$$
G \sim x_{1}^{2}+x_{2}^{2}+(p \delta u+\delta v) \delta v+d_{0}+\sum_{j=1}^{p-1} d_{j}\left(e^{\frac{2 \pi i}{p} a}\right)^{j}(1+j \delta u+\delta v) .
$$

Completing the squares and evaluating the constant piece in the leading order puts $G=0$ in the form

$$
x_{1}^{2}+x_{2}^{2}+x_{3}^{2}+x_{4}^{2}-\mu=0,
$$

with $\mu=\sum_{j=0}^{p-1} d_{j} e^{\frac{2 \pi i}{p} a j}$. Thus, we can choose the flat coordinate $\hat{t}_{a}$ as

$$
\hat{t}_{a} \sim \sum_{j=0}^{p-1} d_{j} e^{\frac{2 \pi i}{p} a j}
$$

Repeating this for all $a=0,1, \ldots, p-1$, we find that the discriminant $\Delta$ to the leading order in $d$ is given by

$$
\Delta \sim \hat{t}_{0} \hat{t}_{1} \cdots \hat{t}_{p-1} \sim \prod_{a=0}^{p-1} \sum_{j=0}^{p-1} d_{j} e^{\frac{2 \pi i}{p} a j}=\operatorname{det}_{i, j}\left(d_{i-j \bmod p}\right) .
$$




\section{$3.2 x_{1}^{2}+x_{2}^{2}+x_{3}^{2}+w^{\prime}\left(x_{4}\right)^{2}+f\left(x_{4}\right)=0$}

Essentially the same analysis applies to the geometry

$$
x_{1}^{2}+x_{2}^{2}+x_{3}^{2}+w^{\prime}\left(x_{4}\right)^{2}+f\left(x_{4}\right)=0,
$$

which has been extensively studied in the context related to gauge theory/matrix model correspondence [19]. Here $w(x)=\frac{1}{n+1} x^{n+1}+\ldots$ and $f$ are polynomials of degrees $n+1$ and $n-1$, respectively. This means that $w^{\prime}(x)^{2}+f_{n-1}(x)=x^{2 n}+\ldots$ is an arbitrary polynomial of degree $2 n$ with unit coefficient of $x^{2 n}$. The non-linear sigma model on the geometry can be realized as the IR limit of the Landau-Ginzburg model with superpotential

$$
W=\Lambda\left(X_{1}^{2}+X_{2}^{2}+X_{3}^{2}+w^{\prime}\left(X_{4}\right)^{2}+f\left(X_{4}\right)\right) .
$$

When all the coefficients in $f$ become small, $n$ conifold singularities appear and $n$ new branches develop. The $i$-th one is characterized by large values of $\lambda$ and $x_{1}=x_{2}=$ $x_{3}=0, x_{4}=a_{i}$ where $w^{\prime}(x)=\prod_{a=1}^{n}\left(x-a_{i}\right)$. We define the $i$-th Coulomb domain to be the place where the scalar field $\lambda$ in the lowest component of $\Lambda$ becomes large, and $x_{4}$ is frozen to $a_{i}$. In this case, the $i$-th Coulomb domain is described by the LandauGinzburg model with $W=s_{i} / X$, where $s_{i}=\int_{A_{i}} \Omega=\int_{A_{i}} d x_{1} d x_{2} d x_{3} d x_{4} / d G$ is the flat coordinate. $A_{i}$ is the $S^{3}$ obtained by deforming the conifold singularity at $x_{4}=a_{i}$. Applying the usual arguments, we get the 't Hooft expansion for the B-model open string on the blow-up of $x_{1}^{2}+x_{2}^{2}+x_{3}^{2}+w^{\prime}\left(x_{4}\right)^{2}=0$. Hence this B-model open string is large $N$ dual to the B-model closed string on $G=x_{1}^{2}+x_{2}^{2}+x_{3}^{2}+w^{\prime}\left(x_{4}\right)^{2}+f\left(x_{4}\right)=0$. This proves the large $N$ duality of the type used in [19].

We find it interesting to look at the deformed conifold case from this point of view. In this case, $w(x)=x^{2} / 2$ and the Landau-Ginzburg superpotential is

$$
W=\Lambda\left(X_{1}^{2}+X_{2}^{2}+X_{3}^{2}+X_{4}^{2}-\mu\right) .
$$

In the previous examples, the effective superpotential $W_{\text {eff }} \sim 1 / X$ are given by applying the Gaussian approximation to the chiral multiplet fields, which is valid when $|\lambda| \gg 1$. In the case of the deformed conifold, this approximation is exact for any $\lambda$ since $W$ is already quadratic in $X_{1}, \ldots, X_{4}$. Thus, we obtain $W_{\text {eff }}=\mu / X$ without any approximation. ${ }^{5}$ Therefore the sigma model on the deformed conifold is equivalent to the Landau-Ginzburg model with the $1 / X$ superpotential [17, 20].

Since the $1 / X$ Landau-Ginzburg model - effective theory in the $C$ phase - is equivalent to the sigma model for the deformed conifold, full topological string amplitudes in this case are computable with worldsheets in pure $C$ phase alone. This means that, from the point of view of the open string dual, perturbative open string Feynman diagrams should not contribute to topological string amplitudes. Indeed, this is consistent

\footnotetext{
${ }^{5}$ The point arose from discussion with Donal O'Connell.
} 
with the fact that the corresponding hermitian matrix model is Gaussian and there are no perturbative contributions [19]. This is a non-trivial check of our worldsheet analysis.

\section{Acknowledgments}

We would like to thank Mina Aganagic, Jaume Gomis, Nick Halmagyi, Kentaro Hori, Amir Kashani-Poor, Yi Li, Marcos Mariño, Donal O’Connell, Christian Roemelsberger, and Cumrun Vafa for useful discussions. This research is supported in part by DOE grant DE-FG03-92-ER40701.

\section{References}

[1] J. M. Maldacena, "The large $N$ limit of superconformal field theories and supergravity," Adv. Theor. Math. Phys. 2, 231 (1998), hep-th/9711200.

[2] R. Gopakumar and C. Vafa, "On the gauge theory/geometry correspondence," Adv. Theor. Math. Phys. 3, 1415 (1999), hep-th/9811131.

[3] R. Dijkgraaf and C. Vafa, "A perturbative window into non-perturbative physics," arXiv:hep-th/0208048.

[4] H. Ooguri and C. Vafa, "Worldsheet derivation of a large $N$ duality," Nucl. Phys. B 641, 3 (2002), hep-th/0205297.

[5] E. Witten, "Phases of $\mathcal{N}=2$ theories in two dimensions," Nucl. Phys. B 403, 159 (1993), hep-th/9301042.

[6] E. Witten, "Some comments on string dynamics," Proceedings of the conference, Strings 95, Los Angeles, California, hep-th/9507121.

[7] M. Aganagic, A. Klemm, M. Marino and C. Vafa, "Matrix model as a mirror of Chern-Simons theory," hep-th/0211098.

[8] K. Hori and C. Vafa, "Mirror symmetry," hep-th/0002222,

[9] M. Aganagic and C. Vafa, "G(2) manifolds, mirror symmetry and geometric engineering," arXiv:hep-th/0110171.

[10] H. Ooguri and C. Vafa, "Knot invariants and topological strings," Nucl. Phys. B 577, 419 (2000), hep-th/9912123. 
[11] J. M. F. Labastida and M. Marino, "Polynomial invariants for torus knots and topological strings," Commun. Math. Phys. 217, 423 (2001), hep-th/0004196.

[12] P. Ramadevi and T. Sarkar, "On link invariants and topological string amplitudes," Nucl. Phys. B 600, 487 (2001) hep-th/0009188.

[13] J. M. F. Labastida, M. Marino and C. Vafa, "Knots, links and branes at large N," JHEP 0011, 007 (2000), hep-th/0010102.

[14] N. Halmagyi and V. Yasnov, "The spectral curve of the lens space matrix model," hep-th/0311117.

[15] N. Halmagyi, T. Okuda and V. Yasnov, "Large $N$ duality, lens spaces and the Chern-Simons matrix model," hep-th/0312145.

[16] D. R. Morrison and M. Ronen Plesser, "Summing the instantons: quantum cohomology and mirror symmetry in toric varieties," Nucl. Phys. B 440, 279 (1995), hep-th/9412236.

[17] D. Ghoshal and C. Vafa, " $c=1$ string as the topological theory of the conifold," Nucl. Phys. B 453, 121 (1995), arXiv: hep-th/9506122.

[18] C. Vafa, "A stringy test of the fate of the conifold," Nucl. Phys. B 447, 252 (1995), hep-th/9505023.

[19] R. Dijkgraaf and C. Vafa, "Matrix models, topological strings, and supersymmetric gauge theories," Nucl. Phys. B 644, 3 (2002), hep-th/0206255.

[20] D. Ghoshal and S. Mukhi, "Landau-Ginzburg model for a critical topological string." In S. R. . Das et. al., ed., "Modern Quantum Field Theory". Proceedings, 2nd International Colloquium, Bombay, India, January 5-11, 1994, http://theory.tifr.res.in/group/colloq/articles.html. 